\title{
Zwei ptolemäische Texte aus der Hamburger Papyrussammlung
}

Mit Abbildungen 4 und 5

\author{
Bärbel Kramer und Dieter Hagedorn (Heidelberg)
}

Zu Wolfgang Müllers Arbeitsgebieten gehören auch die ptolemäischen Papyri aus Tholthis im Oxyrhynchites, deren Edition er im zehnten Band der Berliner Griechischen Urkunden vorgelegt hat. Wir freuen uns, ihm zu seinem 65. Geburtstag die folgende Publikation zweier Urkunden aus demselben Ort widmen zu dürfen. ${ }^{1}$ )

\section{Eingabe an den König}

P. Hamb. Inv. 601

$15,4 \times 16,4 \mathrm{~cm}$

Tholthis

3. Jhdt. v. Chr.

$A b b .4$

Das Fragment stellt knapp die Hälfte des ursprünglichen Papyrusblattes dar. Erhalten ist der linke Teil mit allen drei dazugehörigen Rändern (oben $1,5 \mathrm{~cm}$, unten $4 \mathrm{~cm}$, links $2 \mathrm{~cm}$ ) und offenbar in originaler Höhe. Das Blatt war über die Breite hin einmal zusammengefaltet worden; außer einem dadurch entstandenen Riß, der über die ganze sechste Zeile hinwegreicht, sind auch in den folgenden Zeilen umfangreiche Zerstörungen zu beklagen.

Die Schrift, eine sehr schöne, deutliche Hand des 3. Jhdts. v. Chr., steht quer zu den Fasern, doch auf dem Recto des Blattes; das Verso ist leer. Es ist beobachtet worden, daß die $\dot{\varepsilon}^{v} \tau \varepsilon v^{\prime} \xi \varepsilon l \zeta$ des 3. vorchristlichen Jhdts. fast regelmäßig auf das transversa charta verwendete Recto eines von der Rolle geschnittenen Blattes geschrieben worden sind, so daß die Breite der Eingabe der Höhe der Rolle entspricht; von Ausnahmen abgesehen waren diese Schriftstücke nie weniger als $30 \mathrm{~cm}$, aber auch nie mehr als $34 \mathrm{~cm}$ breit. ${ }^{2}$ ) Offenbar war ein bestimmtes repräsentatives Format für diesen Typ von Bittschrift üblich, wenn nicht gar Vorschrift. Wenn wir bei dem vorliegenden Text die Blattbreite entsprechend der für Z. 1 vorgeschlagenen Ergänzung berechnen, ergibt sich eine Breite von etwa $34 \mathrm{~cm}^{3}$ )

Der Ibiswärter Senychis erhebt Beschwerde gegen den Soldaten Moschion wegen Gewaltanwendung und Diebstahl. Die Einzelheiten des Textes sind verloren; wir

\footnotetext{
1) Wir danken dem Direktor der Staats- und Universitätsbibliothek Hamburg, Herrn Prof. Horst Gronemeyer, für die Publikationserlaubnis und Frau Eva Horváth für die Ubersendung der Photographien und die Möglichkeit, die Originale an Ort und Stelle zu kollationieren.

$\left.{ }^{2}\right)$ O. Guéraud, $E N T E Y \Xi E I \Sigma$. Requêtes et plaintes adressées au roi d'Egypte au $\mathrm{III}^{\mathrm{e}}$ siècle avant J. C., Le Caire 1931, S. XIX - XX.

${ }^{3}$ ) Für den Umfang des vorgeschlagenen Ergänzungstextes war wiederum das in etwa zu erreichende Format unter Berücksichtigung der für die übrigen Zeilen erforderlichen Ergänzungen aussehlaggebend.
} 
erfahren immerhin, daß Moschion die Kühe des Senychis von der Landarbeit auf dem Feld weggeführt hat, sodann bei Senychis zu Hause eingebrochen ist und Geld oder Baumaterial gestohlen hat, das er dann vermutlich mit Hilfe des gestohlenen Gespanns abtransportiert und, wie es scheint, verkauft hat. Die erhaltenen Spuren könnten darauf hinweisen, daß Senychis bereits einmal Anzeige erstattet, Moschion aber auf die daraufhin ergangenen amtlichen Anordnungen nicht reagiert hat. Nun soll der bisher unbekannte Stratege Philippos die Angelegenheit untersuchen. ${ }^{4}$ ) Senychis scheint die Rückgabe seines Eigentums oder Schadenersatz und dazu die Sicherstellung des Utbeltäters zu verlangen.

Ähnlichen Inhalt haben wohl die Eingaben P. Petrie II 31 und BGU VI 1253 gehabt. Im Formular folgt unser Text anscheinend nicht den Parallelen (s. auch den Einzelkommentar).

Eine geographische Angabe ist nicht erhalten. Folgende Gründe scheinen uns aber dafür zu sprechen, daß der Text aus Tholthis im Oxyrhynchites stammt: Moschion ist ein Reiter aus dem Kavallerieregiment des uns wohlbekannten eponymen Offiziers Zoilos, der in zahlreichen Texten aus Tholthis und Umgebung begegnet. ${ }^{5}$ ) Hinzu kommt, daß der Name des Bittstellers Senychis in nur noch zwei Papyri aus dem 3. Jhdt. v. Chr. bezeugt ist, die beide aus Tholthis kommen (s. unten zu Z. 1). Ferner wissen wir, daß es in Tholthis einen Kleros des Moschion gegeben hat (s. zu Z. 2).

Anhand der Daten, die für den eponymen Offizier Zoilos zur Verfügung stehen, läßt sich auch der vorliegende Text zeitlich einordnen, wenn auch nicht genau datieren. Soldaten seiner Truppe sind bisher von 263 bis $229 \mathrm{v}$. Chr. bezeugt, in Tholthis selbst zwischen 258/7 und vielleicht $230^{6}$ ) mit Sicherheit aber bis zum Juni/Juli 231 v. Chr. ${ }^{7}$ ) Als Adressaten der Enteuxis kämen also Ptolemaios II. Philadelphos (283-246 v. Chr.) oder Ptolemaios III. Euergetes I. (246-221 v. Chr.) in Frage.

Die Urkundengattung der Enteuxis ist reichlich bezeugt und durch Untersuchungen

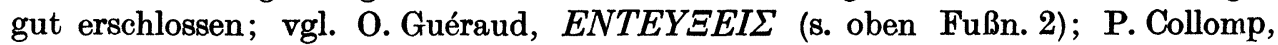
Recherches sur la chancellerie et la diplomatique des Lagides, Paris 1926, S. 51 - 166; A. Di Bitonto, Le petizioni al re, Aegyptus 47, 1967, 5-57. Heranzuziehen sind auch A. Di Bitonto, Le petizioni ai funzionari, Aegyptus 48, 1968, 53-107 und ead., Frammenti di petizioni del periodo tolemaico, Aegyptus 56, 1976, 109-143. Zur Briefform der Eingaben s. J. L. White, The Form and Structure of the Official Petition: A Study in Greek Epistolography, Missoula 1972.

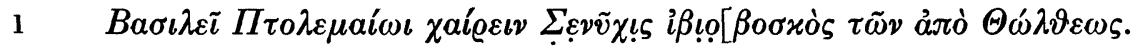
$\dot{\alpha} \delta \iota x о \tilde{v} \mu \alpha \iota$ vं

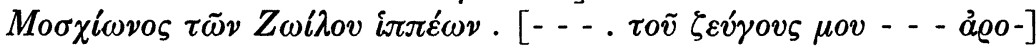

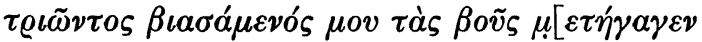

$\left.{ }^{4}\right)$ In der zweiten Hälfte des 3. Jhdts. gelangen die Enteuxeis ohnehin nicht mehr vor den Körig selbst, sondern gehen direkt an die Strategen; vgl. H. J. Wolff, Das Justizwesen der Ptolemäer (Münchener Beiträge 44), München 1962, S. 9-10.

5) Vgl. F. Uebel, Die Kleruchen Ägyptens unter den ersten sechs Ptolemäern, Berlin 1968, S. 135, Fußn. 3; S. 309, Fußn. 5 und öfter.

6) Vgl. Uebel, Die Kleruchen, S. 309, Fußn. 5.

${ }^{7}$ ) Vgl. unten Nr. 2, 17; der Text ist auf den Monat Peritios/Pachons des Jahres 231 v. Chr. datiert. 


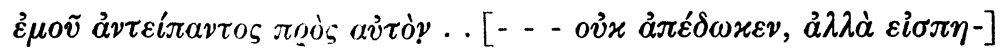

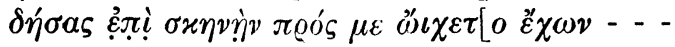

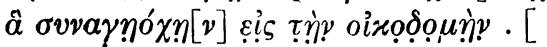

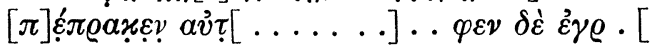

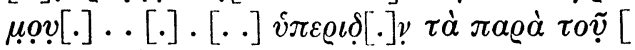

$\pi \varrho \circ \sigma \tau \alpha \dot{\xi}[\alpha \iota] \Phi[\iota] \lambda i \iota \pi \omega \iota \tau \tilde{\omega} \iota \sigma \tau \varrho \alpha \tau \eta \gamma \tilde{\omega} \iota[$

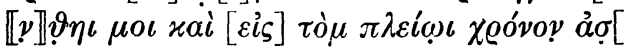

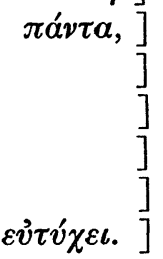

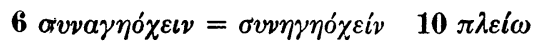

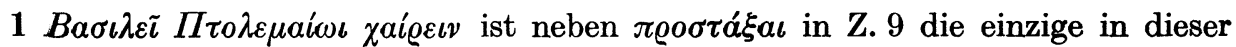
Enteuxis enthaltene stereotype Wendung; vgl. dazu Aegyptus 47, 1967, 11. Zur Frage des Adressaten s. o. die Einleitung S. 10.

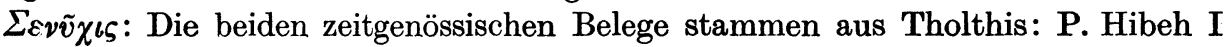
61, 6 (245/4; zur Herkunft der Texte der Mumie A 9 vgl. Uebel, Die Kleruchen, S. 300, Fußn. 3) und BGU XIV 2394, 7. [10] (216/5); später begegnet der Name noch einmal in P. Tebt. I $82=$ W. Chr. 232, 43 (115 v. Chr.; Magdola). Varianten sind $\Sigma \varepsilon v o \tilde{\chi} \chi \iota \varsigma$ und $\Sigma \varepsilon v \tilde{\omega} \chi \iota \varsigma$; vgl. die Namenbücher und PPtol. VII s. vv.

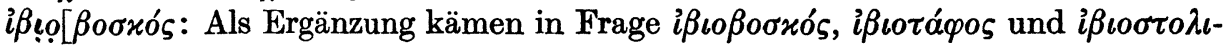
$\sigma \tau \eta \dot{s}$. Wir haben $i \beta \iota$ $\beta$ oбxós ergänzt, weil dieser Beruf im 3. Jhdt. v. Chr. erheblich

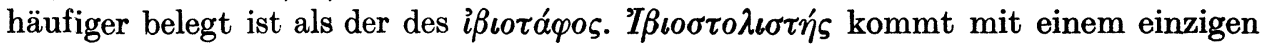
Beleg erst aus römischer Zeit (P. Fay. 246; 1./2. Jhdt. n. Chr.) kaum in Betracht.

Eine Sammlung und Untersuchung der Testimonien für diese Ämter ist vorgelegt worden von A. Traversa, Ibiotáphoi, Ibioboskoí, e un cartonage inedito della collezione osloense SO 36, 1960, 4-64; vgl. dazu auch W. Clarysse und J. Quaegebeur, Ibion, Isieion and Tharesieion in two Oslo Papyri, SO 57, 1982, 69-85.

In der Priesterhierarchie nimmt der Ibiswärter eine sehr niedrige Stellung ein; er steht noch unter dem Ibisbalsamierer. Er bewirtschaftet, wie man auch hier bei Senychis sieht, Land, teils zum Anbau von Futter für die Ibisse ( $\imath \beta i \omega v \tau \varrho o \varphi \eta ́)$, daneben sicher auch zum eigenen Bedarf. Aus P. Fouad 16 geht hervor, daß es im Oxyrhynchites im 2. Jhdt. v. Chr. vier Ibisheiligtümer gegeben hat; eines davon scheint, wie unser Text zeigt, in Tholthis bereits im 3. Jhdt. v. Chr. bestanden zu haben.

[ $\tau \tilde{\omega} \nu$ ảjò $\Theta \dot{\omega} \lambda \vartheta \varepsilon \omega \varsigma]$ : Zur Herkunft der Enteuxis s. oben die Einleitung S. 10. Von den drei Dörfern namens Tholthis in der Unteren, der Oberen und der ThmoisephoToparchie des Oxyrhynchites darf im 3. Jhdt. v. Chr. immer mit demjenigen in der

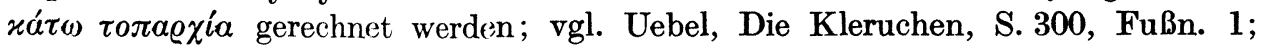
P. Pruneti, Note di toponomastica, Aegyptus 59, 1979, 101-107; ead., I centri abitati dell'Ossirinchite (Papyrologica Florentina 9), Firenze 1981, S. 58-63; A. Calderini-S. Daris, Dizionario dei nomi geografici e topografici dell'Egitto greco-romano II, Milano 1975, S. 299 f.

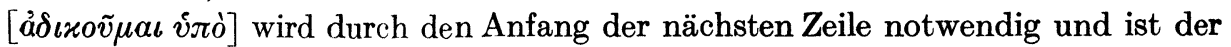
häufigste Beginn einer Enteuxis; vgl. Aegyptus 47, 1967, 14.

2 Der Soldat Moschion war bisher unbekannt. Im Zusammenhang mit Tholthis

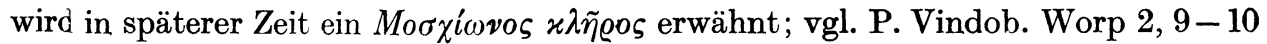

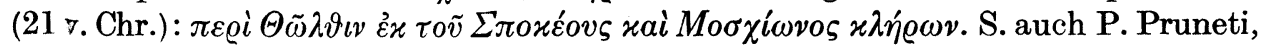

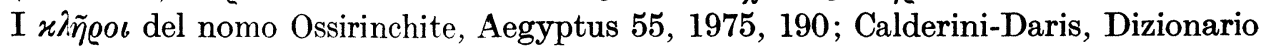
III, 1982, S. 297. 
$\tau \tilde{\omega} v Z \omega i \lambda_{o v} i \pi \pi \varepsilon ́ \omega v$. [: Zu Zoilos s. oben die Einleitung S. 10 mit Fußn. 5 und

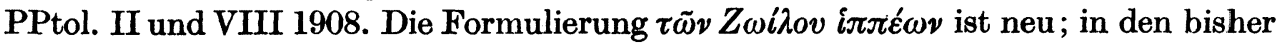
bekannten Texten lautete die Personalangabe der Truppenangehörigen entweder: Name + Ethnikon + Rang + $\tau \tilde{\omega} \nu$ Z $\omega$ i $\lambda$ ov oder: Name + Rang $+\tau \tilde{\omega} \nu Z \boldsymbol{Z} \omega \boldsymbol{i} \lambda o v$ oder: Name + Ethnikon $+\tau \tilde{\omega} v Z \boldsymbol{Z} \omega i \boldsymbol{\lambda} o v$, oder: Name $+\tau \tilde{\omega} v Z \boldsymbol{Z} \omega \mathbf{i} \lambda o v+$ Rang. Unter den

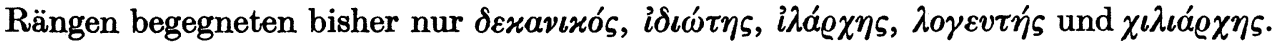
Es ist kein Fall bekannt, wo das Ethnikon auf den Namen des Offiziers folgt.

Wenn das hier auf $i \pi \pi \dot{\varepsilon} \omega v$ folgende Wort noch zur Personenbeschreibung des Mo-

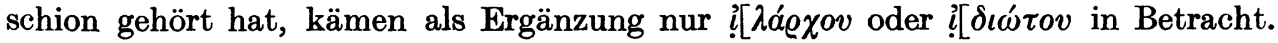
Die erhaltene senkrechte Haste scheint uns zwar am besten zu einem $\varkappa$ zu passen, doch sind auch $\mu$ oder $\iota$ nicht vollends auszuschließen; ein Beispiel für besonders

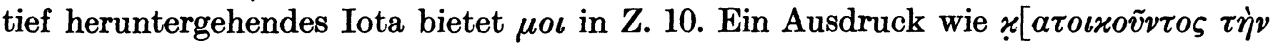

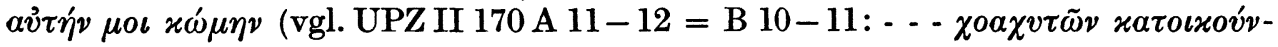

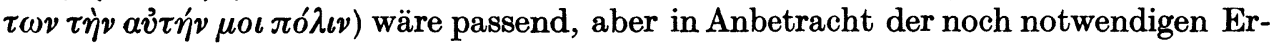
gänzungen zu lang. $x[\alpha \tau o i x \omega \nu$ läge nahe, ist jedoch abgesehen davon, daß es immer

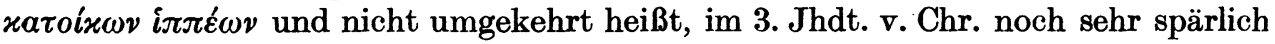
belegt (vgl. Uebel, Die Kleruchen, S. 33, Fußn. 3) und sollte daher hier besser nicht ergänzt werden; $\chi[\lambda \eta \varrho o v ́ \chi o v$ entfällt, weil der Ausdruck im Oxyrhynchites in diesem Zusammenhang nicht verwendet wird; die einzige Ausnahme ist die Verbindung

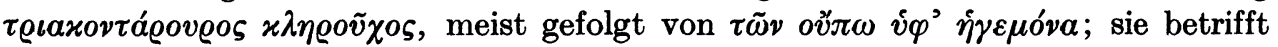
also nur Fußsoldaten (vgl. Uebel, Die Kleruchen, S. $380 \mathrm{f}$.).

Wollte man das anfangende Wort zum Folgenden ziehen, ergäben sich wohl weniger Möglichkeiten. Es beginnt ja die Schilderung des Tathergangs, die durch irgendeine adverbiale Bestimmung eingeleitet worden sein muß, da die andere Möglichkeit, das Partizip im Genitivus absolutus, anscheinend erst am Zeilenende beginnt. Man

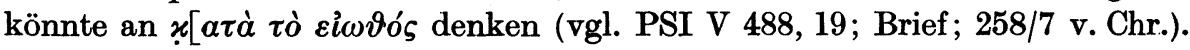

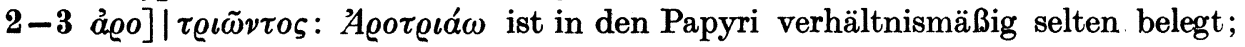
neben SB V 7758, 25 (497 n. Chr.), P. Flor. III 383, 89 (232 n. Chr. ff.) und den fragmentarischen ptolemäischen Zeugnissen PSI VI 661, 5 und P. Cairo Zenon IV 729, 5 (beide 3. Jhdt. v. Chr.) begegnet es nur noch in einer Eingabe, der anscheinend eine ähnliche Situation zugrunde liegt, P. Petrie III 31, 4 ff. (3. Jhdt. v. Chr.): $\tau \tilde{\eta} \iota \xi$

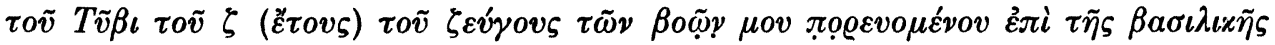

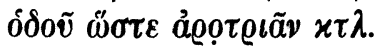

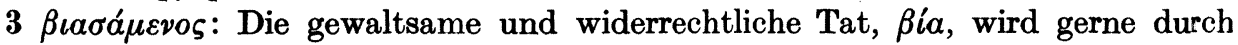
$\beta \iota a ́ \zeta о \mu \alpha \iota$ im Partizip zur Verstärkung des Hauptverbs ausgedrückt; gelegentlich erscheint $\beta \iota a ́ \zeta o \mu \alpha \iota$ auch in Komposita und als Hauptverb mit entsprechender Bedeutung; vgl. Aegyptus 47, 1967, 27f. und 48, 1968, 79; als Beispiel s. BGU VI 1253, 8 (zitiert unten).

$\mu[\varepsilon \tau \dot{\eta} \gamma a \gamma \varepsilon v: M \varepsilon \tau \alpha \dot{\gamma} \omega \omega$ ist das gebräuchliche Verb zur Bezeichnung der Überführung von Tieren von einem Platz zum anderen, häufig von einer Weide auf die andere;

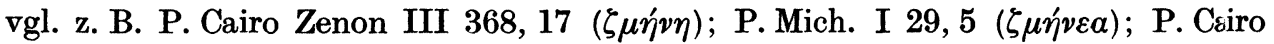

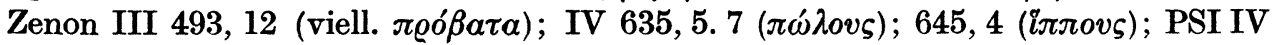

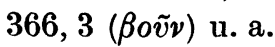

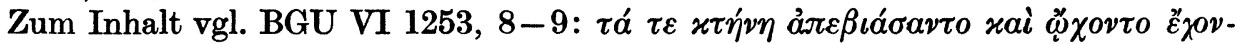
$\tau \varepsilon \varsigma$.

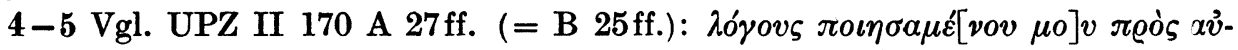

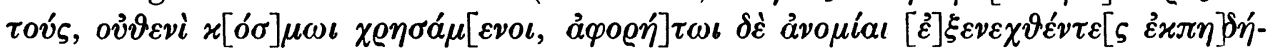

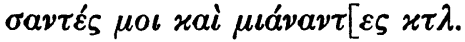


$\varepsilon i \sigma \pi \eta] \mid \delta \eta ́ \sigma \alpha \nu \tau \varepsilon \varsigma$ : Ergänzt nach P. Petrie II 32, 17-18 (zit. unten zu Z. 5). Die Eingaben sind reich an Komposita wie $\dot{\varepsilon} \varkappa-,, \dot{\varepsilon} \mu-$, $\dot{\varepsilon} \pi \iota-$, $\varepsilon \dot{\imath} \sigma-, \pi \varrho o \sigma \pi \eta \delta a ́ \omega$ u. a. (vgl. Aegyptus 48, 1968, 76), wo auch immer von Ưberfall und Einbruch die Rede ist.

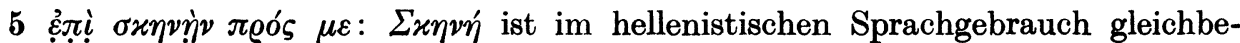
deutend mit „Haus“, wohl eine Folge des Soldatenlebens in den asiatischen Feld-

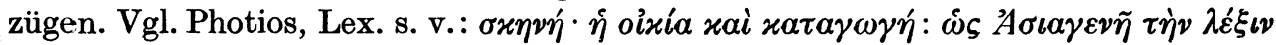

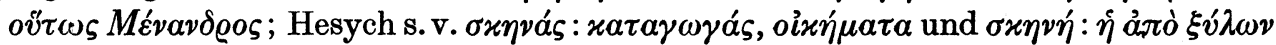

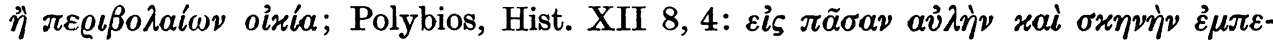

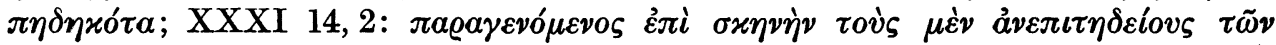

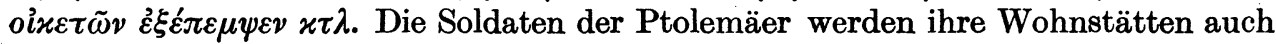
dann noch mit ,Zelt“ bezeichnet haben, als sie schon in festen Häusern seßhaft waren. Dies zeigen auch die Papyri; neben dem vorliegenden Testimonium sei auf SB XII 10782 (= BASP 7, 1970, 40 = P. Hibeh I 129 descr.) verwiesen (247/6 v. Chr.),

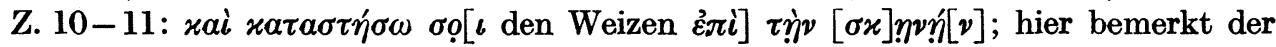

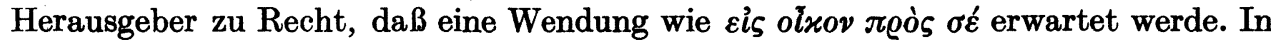
demselben Sinne ist der Ausdruck in einem noch unveröffentlichten prodomatischen Pachtvertrag, P. Hamb. Inv. 401, gebraucht, der aus derselben Zeit stammen

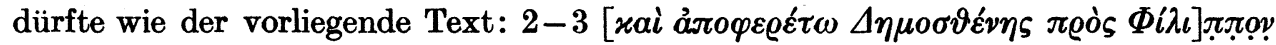

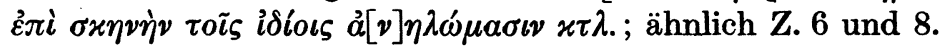

Die Verstärkung durch die Angabe der Person findet sich auch beispielsweise in

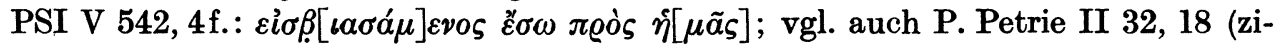
tiert im nächsten Abschnitt).

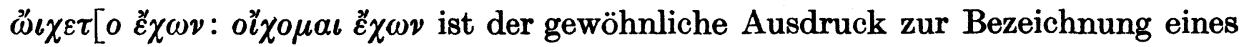
Diebstahls; davor ist ein Kompositum von $\pi \eta \delta a ́ \omega$ beliebt. Vgl. BGU VI 1253, 9-10 (zit. aben zu 3); P. Coll. Youtie I 7, 6-7; P. Frankfurt 3, 20 mit Testimoniensammlung im Kommentar; Aegyptus 47, 1967, $29 ; 48,1968,81 ; 56,1976,125$. Als Beispiel

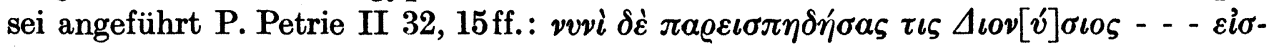

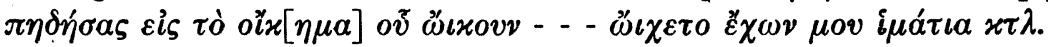

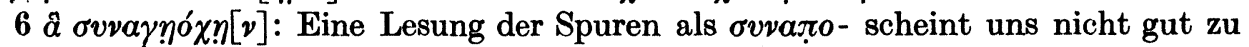
sein, weil die Beine des Pi dann stärker nach innen eingebogen sein müßten, als die vorhandenen Hasten es sind. Wenn hier eine Verbform gestanden hat, dann muß sie augmentiert gewesen sein; ein Augment ist aber nicht erkennbar. Die einzige in Frage kommende Möglichkeit ist ein Plusquamperfekt, bei dem in gewissen Fällen das Augment vor der Reduplikation fehlen kann; dies läßt sich besonders bei der attischen Reduplikation nachweisen.

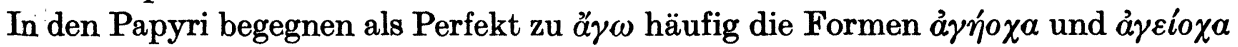

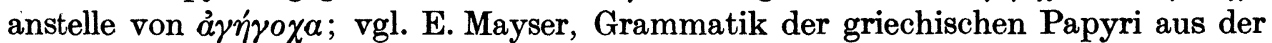
Ptolemäerzeit I 2, Leipzig - Berlin ${ }^{2}$ 1938, S. 104- 105. Hier dürfte aus Platzgründen und wegen der oben angeführten Erwägungen das Plusquamperfekt gestanden

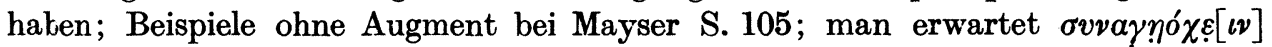
am Ende scheint jedoch eine gerade Haste erhalten zu sein, die eher zu Eta paßt; die Schreibung von $\eta$ für $\varepsilon \iota$ ist eine geläufige Erscheinung.

Inhaltlich ergibt sich ein guter Sinn; es wird gesagt, daß Moschion Dinge mitgenommen hat, die Senychis für ein beabsichtigtes Bauvorhaben zu Hause angesamme.t hat; dies kann Geld sein, es können aber auch Baumaterialien wie Holz oder Steine sein, zu deren Abtransport Moschion sich vorsorglich die Kühe angeeignet

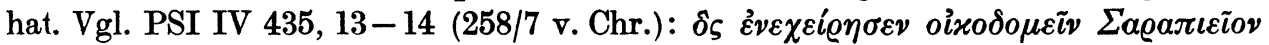




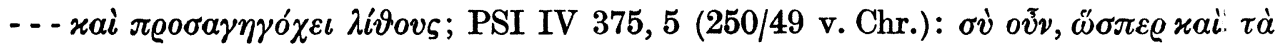

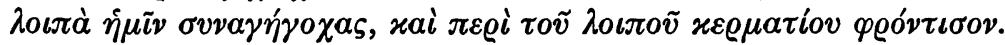

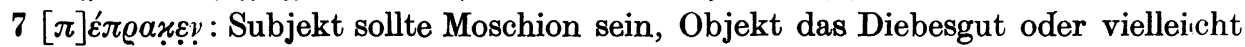

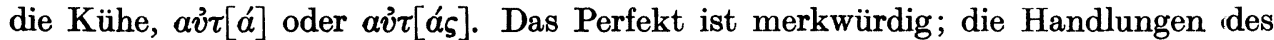
Moschion sind ansonsten im Aorist geschildert. Vielleicht hat Senychis später gehört, daß Moschion die Sachen verkauft hat.

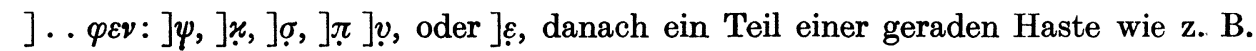
von 6 .

$\delta \dot{\varepsilon} \dot{\varepsilon} \gamma \varrho .\left[:\right.$ Der Anschluß des $\delta \dot{\varepsilon}$ ist rätselhaft. $\dot{\varepsilon} \gamma \varrho$.[: Nach $\varrho$ kaum $\alpha$, vielleicht $\eta_{l}$,

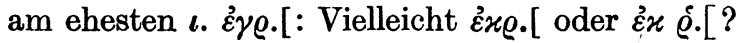

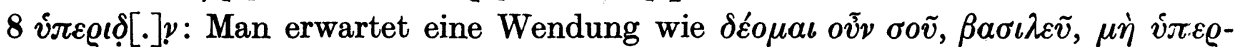

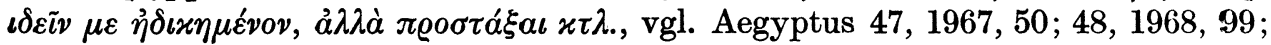
56, 1976, 137. Derartiges hat hier aber, wie der Kontext trotz der Verluste erkennen läßt, nicht gestanden. Unterhalb der Zeile ist keine Spur von der Haste eines Iota von - $\varepsilon \iota v$ zu erkennen; wenn das Iota auch nie übermäßig lang heruntergezogen wird, so müßte es doch mindestens genau so tief heruntergereicht haben wie das $\nu$. Folglich ist anzunehmen, daß der Papyrus $v \pi \varepsilon \varrho \iota\left[\omega^{\prime}\right]$. gehabt hat. Man könnte an eine Formu-

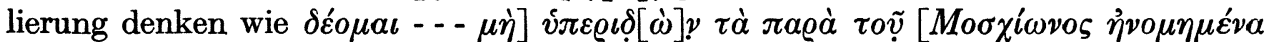

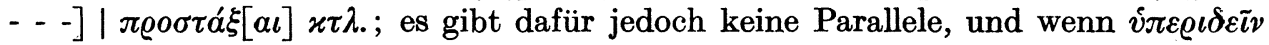
vom König gesagt wird, ist die Formel festgelegt.

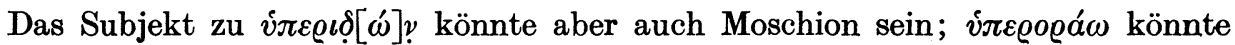

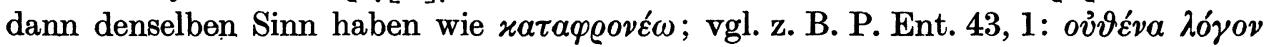

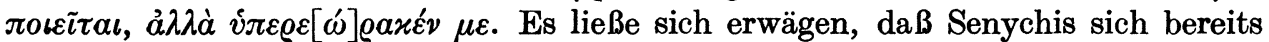
früher an die Behörden gewandt, aber Moschion deren Anordnungen ignoriert hat,

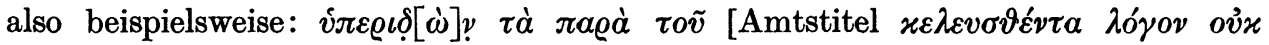

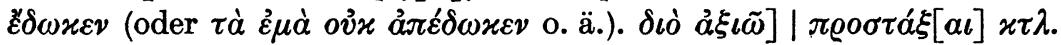

9 Der Stratege Philippos war bisher nicht bekannt. Zu den Strategen des Oxyrhynchites in ptolemäischer und römischer Zeit vgl. zuletzt J. E. G. Whitehorne, A Checklist of Oxyrhynchite Strategi, ZPE 29, 1978, $127 \mathrm{ff}$. Zur Erweiterung der Kompetenz der Strategen vom militärischen Bereich auf die zivile Administration der Gaue, die wohl unter Euergetes eingeführt wurde, vgl. G. Bastianini, Lykomedes, Krisippos, Nikanor: Strateghi nell'Herakleopolites e Oxyrhynchites, Anagennesis 1, 1981, 161-168, bes. 163, Fußn. 11.

10 Am Anfang der Zeile eine Korrektur; anscheinend ist $\vartheta$ in $v$ hineingeschrieben worden. Danach sicher $\eta \iota$. Man darf annehmen, daß Senychis hier Anspruch auf Rückgabe der gestohlenen Sachen erhebt oder auf Schadenersatz besteht; es bieten

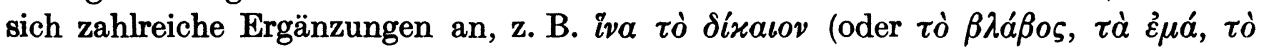

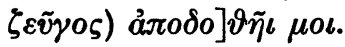

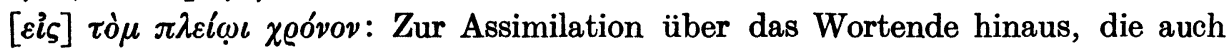
unten in Nr. 2, $19 \mathrm{zu}$ beobachten ist, vgl. E. Schwyzer, Griechische Grammatik I, München ${ }^{2} 1953$, S. 407 f.; E. Mayser - H. Schmoll, Grammatik der griechischen Papyri aus der Ptolemäerzeit I 1, Berlin ${ }^{2} 1970$, S. 203.

Zum hybriden adskribierten Iota s. W. Clarysse, Notes on the use of the iota adscript in the third century B. C., CE 51, 1976, 165 mit Verweis auf P. Cairo Zenon IV 742, 26; vgl. auch unten zu Nr. 2, 3.

$\alpha \sigma[$ : Das Sigma ist sehr klein; vgl. das zweite Sigma von Moơíwvos in Z. 2 und

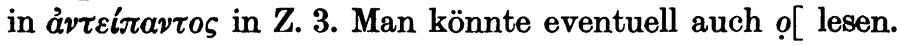


Für den Inhalt dieser Zeilen lassen sich wiederum verschiedene Vermutungen anstellen. In den Parallelen begegnet der Gedanke, daß das gestohlene Gut sicher-

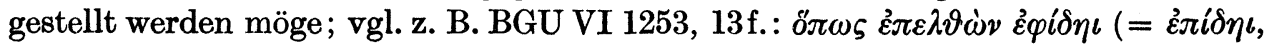

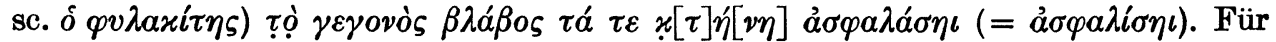
eine entsprechende Ergänzung scheint im vorliegenden Fall der Hinweis auf die $\mathrm{Zu}$ kunft nicht zu passen.

Möglich ist auch, daß Senychis für die Zukunft vor weiteren Anschlägen des Moschion sicher sein und diesen deshalb in Gewahrsam wissen möchte. Vgl. z. B.

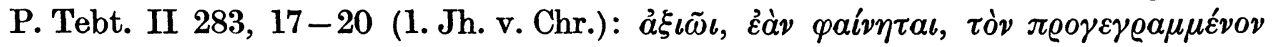

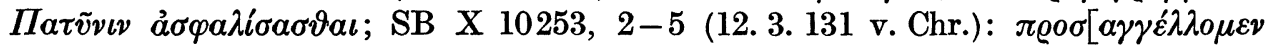

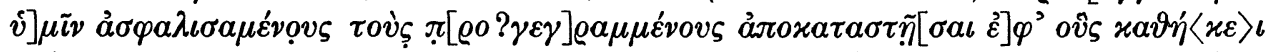

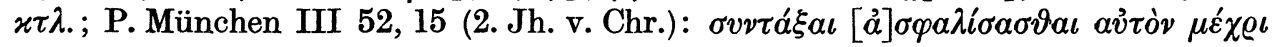

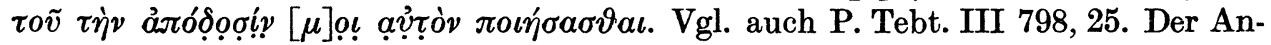
spruch, auch für die Zukunft vor Missetaten geschützt zu sein, kommt deutlich aber nur in einer Eingabe aus frührömischer Zeit zum Ausdruck, P. Oxy. XIX

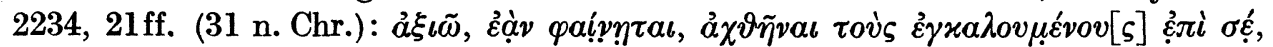

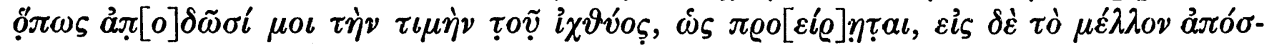

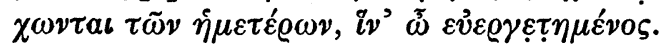

Letztlich ist auch nicht auszuschließen, daß der Gedanke, in Zukunft die Gerechtigkeit und Barmherzigkeit des Königs zu genießen, hier gestanden hat, wie

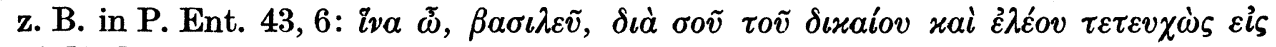

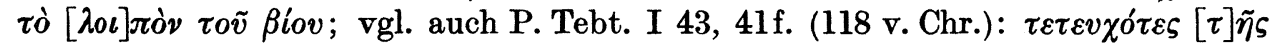

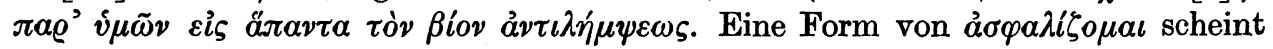
uns aber am ehesten als Ergänzung angebracht.

Eine Rekonstruktion des Textes soll veranschaulichen, wie der Gedankengang gewesen sein könnte; für die Ergänzungen wurden im wesentlichen die oben angeführten Parallelen benutzt:

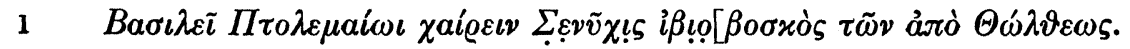
$\dot{\alpha} \delta \iota x o \tilde{v} \mu \alpha \iota$ vंगं ]

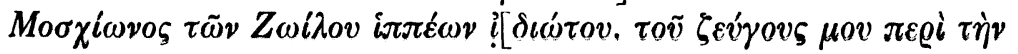
$x \omega \dot{\mu} \mu \eta \nu$ ả $\varrho-]$

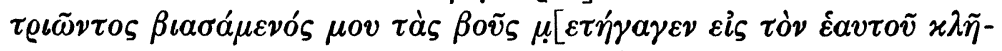
$\varrho o v . \delta \iota \dot{\alpha} \tau o \tilde{v} \tau o]$

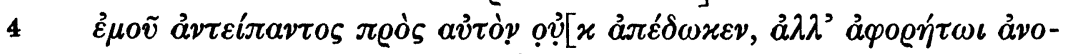

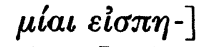

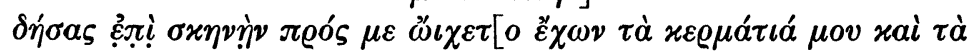

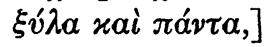

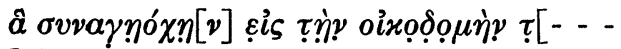

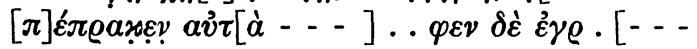

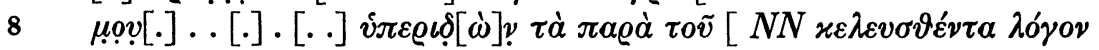
ov่x है $\delta \omega x \varepsilon v . \dot{a} \xi \iota \tilde{\omega}$ oṿv]

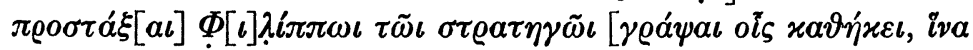

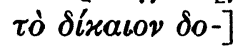

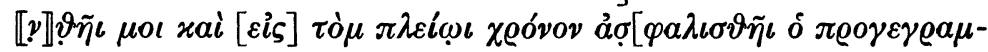
$\mu \varepsilon ́ v o s . \varepsilon v ̉ \tau v ́ \chi \varepsilon \iota$. 


\section{Utbersetzung}

Dem König Ptolemaios Grüße von Senychis, Ibiswärter derer aus Tholthis.

Ich erleide Unrecht von Moschion, einem (einfachen Soldaten) aus der Kavallerietruppe des Zoilos. (Als nämlich mein Gespann in der Gegend des genannten Dorfes) pflügte, führte er unter Gewaltanwendung meine Kühe weg (zu seinem Landlos). Als ich (daher) bei ihm Protest erhob, (gab er sie mir nicht zurück, sondern) brach (mit unerträglicher Frechheit) bei mir zu Hause ein und entkam mit (meinem Geld, dem Bauholz und allem), was ich für den Bau (meines - - -) angesammelt hatte - . hat er verkauft - - - und er mißachtete (die Anweisungen, die ihm von dem Beanten NN gegeben worden waren, und legte keine Rechenschaft ab. Deshalb beantrage ich), den Strategen Philippos zu beauftragen, (an die zuständigen Personen zu schreiben, damit mir mein Recht gegeben und der Obengenannte) für die Zukunft (in Gewahrsam genommen werde. Lebe wohl).

\section{Gelddarlehen}

P. Hamb. Inv. 676

$16 \times 11,7 \mathrm{~cm}$

Tholthis

17. 6. -16.7. 231 v. Chr.

Abb. 5

In Form einer Sechszeugen-Doppelurkunde ${ }^{8}$ ) kommen der einfache Soldat Menandros und der Zivilist Ammonios über die Modalitäten eines Darlehens von 100 Drachmen überein, das Menandros dem Ammonios gewährt hat. Die Abmachungen halten sich ganz im Rahmen des aus den Parallelen (s. u.) Bekannten; allein die Tatsache, daß keine feste Laufzeit für das Darlehen vereinbart wird, sondern Ammonios dem Menandros das Kapital zehn Tage nach der Aufkündigung durch Menandros zurückzahlen soll, ist eines besonderen Hinweises wert (vgl. den Einzelkommentar zu Z. 21-22).

Der Papyrus ist sowohl oben wie auch unten unvollständig. Links fehlen von den Zeilenanfängen jeweils 1-2 Buchstaben, dagegen ist rechts in Höhe der Zeilen 2-13 kein Verlust zu beklagen; von Z. 13 an werden die Zeilenenden jedoch durch einen bogenförmig von rechts oben nach links unten verlaufenden Schnitt in zunehmendem Maße verkürzt. Dieser Schnitt wie auch die Verstümmelung an den Zeilenanfängen könnten dem Blatt bei seiner Verarbeitung $\mathrm{zu}$ Mumienkartonage zugefügt worden sein, während der Textverlust am oberen Ende eher durch Bruch als durch einen Schnitt zustande gekommen ist.

Vom Wortlaut des Vertrags sind die letzten fünf Zeilen des Innentextes der Doppelurkunde und die ersten 14 Zeilen ihres Außentextes zumindest in Spuren erhalten. Zwischen Innentext und Außentext hatte der Schreiber ein Spatium von etwa $3 \mathrm{~cm}$ Höhe freigelassen. In diesen Freiraum sind von späterer Hand fünf von uns hier nicht transkribierte Zeilen gesetzt worden, die mit dem Darlehen nichts zu tun haben, sondern vermutlich zu einem von uns hier ebenfalls nicht mitgeteilten Text gehören, der sich auf der Rückseite des Blattes findet. Diese spätere Hand schreibt zwar in demselben Stil, aber etwas größer und viel unregelmäßiger als der Schreiber des Darlehensvertrags.

${ }^{8}$ ) Vgl. zu Geschichte, Form und Bedeutung der Sechszeugenurkunde zuletzt H. J. Wolff, Das Recht der griechischen Papyri Ägyptens in der Zeit der Ptolemaeer und des Prinzipats, Bd. II, München 1978, S. 57-80. 
Der Vertragstext ist quer zu den Fasern geschrieben, steht aber dennoch auf der Rektoseite des Papyrusblattes, welches also transversa charta benutzt ist. Diese Anordnung findet sich bei den Sechszeugen-Doppelurkunden besonders der früheren Ptolemäerzeit recht häufig. ${ }^{9}$ )

An Spezialliteratur zum Darlehen im griechisch-römischen Ägypten braucht über diejenige hinaus, die Wolfgang Müller in seiner Einleitung zu BGU X 1961 aufgeführt hat, nichts genannt zu werden. Wir beschränken uns hier darauf, eine Liste der Parallelurkunden aus dem Oxyrhynchites und dem 3. Jh. v. Chr. zusammenzustellen. Es sind uns folgende bekannt geworden:

P. Ross. Georg. II $1+2+$ P. Ien. Inv. 1007 (SB XII 11058) ${ }^{10}$ ); 244 v. Chr.

SB XII 11059; 244 v. Chr.

P. Hibeh II 261 und $262 ; 239$ v. Chr.

BGU VI 1273 = BGU XIV 2395; 222/1 v. Chr.

BGU VI 1274; 218/7 v. Chr.

BGU VI 1276; $215 / 4$ v. Chr.

BGU X 1960; 214/3 v. Chr.

BGU X 1961; 213/2 v. Chr.

BGU X 1962 = BGU XIV 2396; 213/2 v. Chr.

BGU X 1963; 212/1 v. Chr.

BGU X 1965; 212/1 v. Chr.

BGU X 1966; Zeit des Euergetes I. (?)

BGU X 1967; Zeit des Epiphanes.

$\mathrm{Zu}$ vergleichen ist auch das Darlehen mit Wohnungsantichrese in P. Hamb. II 190 = BGU X 1964 aus der Zeit des Philopator.

Spuren

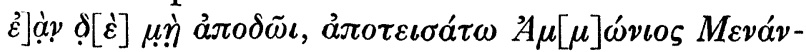

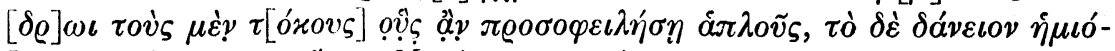

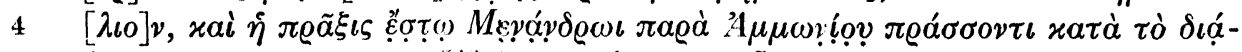

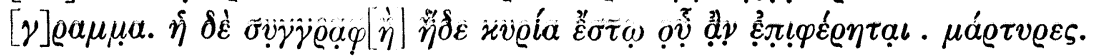

(Zu Z. 6-11 vgl. die Einleitung)

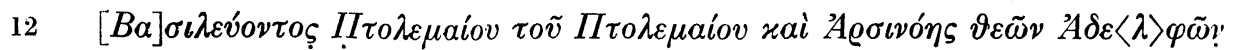

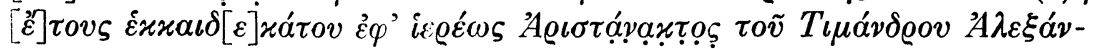

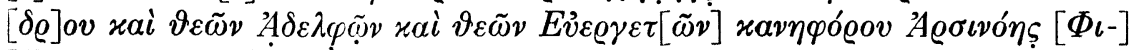

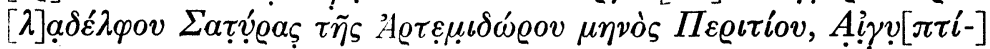

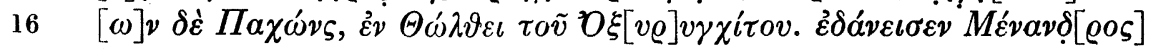

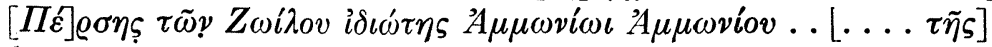

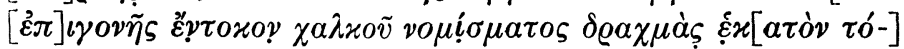

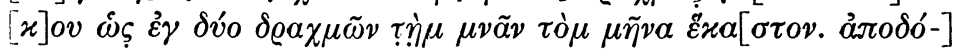

9) Vgl. E. G. Turner, The Terms Recto and Verso. The Anatomy of the Papyrus Roll (Papyrologia Bruxellensia 16), Bruxelles 1978, S. 34.

10) Jgl. F. Uebel, Jenaer Kleruchenurkunden, APF 22/23, 1974, $101-109$. 


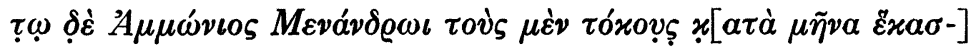

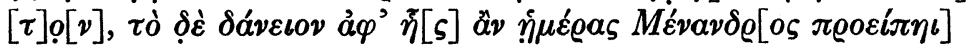

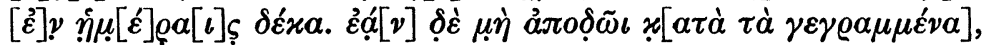

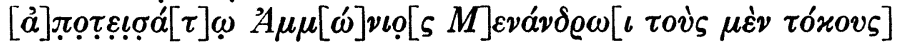

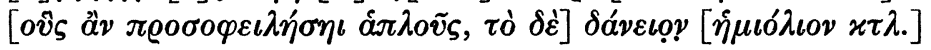

\section{Spuren}

2-4 = 22-24 Die Formulierung der Strafbestimmungen für den Fall, daß das Darlehen nicht rechtzeitig zurückgezahlt wird, stimmt wörtlich mit der in BGU X 1961, 7-9 überein. Sie besagt, daß die Zinsen in einfacher Höhe (nämlich mit 2\% monatlich; s. unten zu Z. 18-19) auch in der Verzugszeit weiterhin berechnet werden sollen, während das Kapital mit einem Aufschlag von $50 \%$ zurückgezahlt werden muß; vgl. W. Müller zu BGU X 1961, 8-9 und jetzt ausführlich W. Schäfer zu P. Köln V 218, 11- 13 (S. 136-138).

$3 \pi \varrho \circ \sigma o \varphi \varepsilon \iota \lambda \eta \dot{\sigma} \sigma$ : Während der Schreiber nach $\omega$ stummes Jota noch regelmäßig adskribiert, hat er es hier nach $\eta$ ausgelassen. Das entspricht der allgemeinen Beobachtung, daß die Empfindung für das Vorhandensein des $\iota$ bei $\eta \iota$ früher abhanden gekommen ist als bei $a \iota$ und $\omega \iota$. Vgl. E. Mayser-H. Schmoll, Grammatik der Griechischen Papyri aus der Ptolemäerzeit I 1, Berlin ${ }^{2} 1970$, S. 96-117; W. Clarysse, Notes on the use of the iota adscript in the third century B. C., CE 51, 1976, 150166.

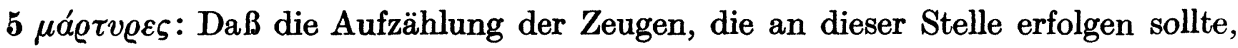
unterbleibt, hat eine Parallele bereits in P. Cornell 2 aus dem Zenonarchiv vom Jahre 248 v. Chr. Man trifft dieselbe Erscheinung sodann in mehreren Verträgen aus Tholthis aus der Zeit um 216-212 v. Chr. an, was H. J. Wolff, Das Recht (s. Fußn. 8) S. 64f. mit dem Prozeß der Verkümmerung des Innentexts der Sechszeugenurkunde in Zusammenhang bringt, der etwa um dieselbe Zeit beginnt (die Belege ebenda S. 64, Fußn. 45; BGU X 1962 = BGU XIV 2396 ist jedoch ein Blankovertrag, der möglicherweise nie vollzogen wurde, und sollte daher besser hier nicht genannt werden; vgl. ferner BGU XIV 2383; 2384; 2393; 2394; 2397; 2398 und 2399). Aus Tholthis ist der vorliegende Text das früheste bisher bekannte Beispiel; er läßt Zweifel daran aufkommen, ob Wolff mit seiner Erklärung das Richtige getroffen hat. Vgl. zu der Frage auch F. Uebel, Ein neues Zeugnis für das Agoranomion von Oxyrhynchos im 3. Jahrhundert v. u. Z. (P Berlin 11803), in: Festschrift zum 150jährigen Bestehen des Berliner Ågyptischen Museums, Berlin 1974, S. 441-452, hier S. 445.

13-15 Die Namen der alexandrinischen eponymen Priester des 16. Regierungsjahres von Ptolemaios III. Euergetes I. $=232 / 1$ v. Chr. waren bisher nur in demotischer Transkription aus dem noch unedierten P. dem. Brit. Mus. 10380 bekannt; vgl. dazu zuletzt W. Clarysse-G. van der Veken, The Eponymous Priests of Ptolemaic Egypt, P. Lugd. Bat. XXIV, Leiden 1983, S. 12 unter Nr. 59. Bei der Entzifferung der demotischen Zeichen bzw. bei ihrer Transposition ins Griechische sind der Forschung jedoch, wie man jetzt erkennen kann, zum Teil Fehler unterlaufen. So war der Name des Alexanderpriesters im Demotischen bisher mit 3rystwnkws

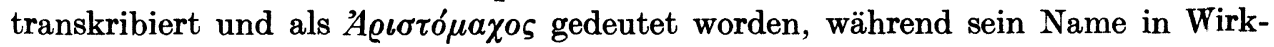

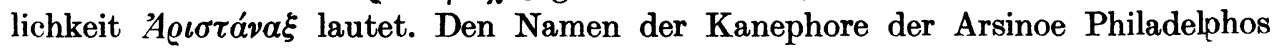




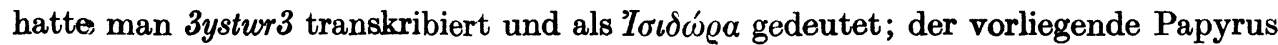
zeigt, daß statt dessen $\Sigma \alpha \tau v ́ \varrho \alpha$ richtig ist. Die Namen der Väter beider Priester waren auch aus dem Demotischen schon korrekt erschlossen worden.

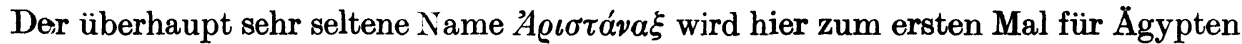
bezeugt.

15 - 16 Der Monat Pachons des 16. Regierungsjahres des Euergetes I. erstreckte sich, in julianische Daten umgerechnet, vom 17. Juni bis zum 16. Juli 231 v. Chr.; vgl. die Umrechnungstabellen bei T. C. Skeat, The Reigns of the Ptolemies (Münchener Beiträge 39), 2. Aufl. München 1969. Die Probleme, vor die im Gegensatz zum ägyptischen Kalender der makedonischen Kalender und Doppeldatierungen unter Euergetes die Forschung noch stellen, diskutiert ausführlich A. E. Samuel, Ptolemaic Chronology (Münchener Beiträge 43), München 1962, S. 75 - 105. Eine knappe Darstellung der verschiedenen, nebeneinander existierenden Datierweisen und außerordentlich praktische, oft aber hypothetische Konkordanztafeln findet man jetzt bei P. W. Pestman u. a., A Guide to the Zenon Archive, A (P. Lugd. Bat. XXI A), Leiden 1981, S. 215 - 265. Das vorliegende Doppeldatum ist zur Klärung der Situation wenig hilfreich, da keine Tagesdaten genannt werden. Immerhin wird Pestmans Konkordanz (a. a. O. S. 257) insofern bestätigt, als nach Ausweis des Papyrus der Monat Peritios sich wirklich mit dem Pachons überschnitt; nach Pestmans Rekonstruktion sollte er vom 29. Pharmuthi bis zum 27. Pachons gedauert haben. Das trifft jedoch nur dann zu, wenn im 16. Regierungsjahr der Schaltmonat noch nach dem Hyperberetaios und nicht, wie es in der Konkordanz als Alternativlösung erwogen und wie es im 20. Regierungsjahr erstmals bezeugt ist, nach dem Peritios eingeschoben worden ist; vgl. dazu Pestman a. a. O. S. 218. Es scheint nun, als könne die Änderung der Praxis erstmals im 18. Regierungsjahr stattgefunden haben.

16 हैं $\Theta \dot{\alpha} \lambda \vartheta \varepsilon \iota$ : Vgl. oben zu Nr. 1, 2.

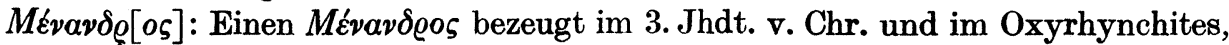
möglicherweise sogar in Tholthis, auch BGU XIV 2387; vgl. ebendort zu Z. 1.

$17 \tau \tilde{\omega} \nu Z \omega i \lambda o v$ : Vgl. zu diesem eponymen Offizier oben zu Nr. 1, 2.

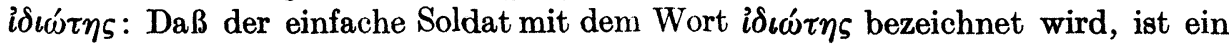
Charakteristikum des Oxyrhynchites; vgl. F. Uebel, Die Kleruchen (s. oben Fußn. 5), S. 380.

Ammonios, der Sohn des Ammonios, war aus anderen Papyri aus Tholthis anscheinend noch nicht bekannt.

Zur Bezeichnung der Personen (Name, Ethnikon, militärischer Rang bei Soldaten; Name, Vatersname, Ethnikon $+\tau \tilde{\eta} \varsigma \dot{\varepsilon} \pi \iota$ yov $\tilde{\eta} s$ bei nicht im Militärdienst stehenden Kleruchensöhnen) vgl. die „Nomenklaturregel“ bei Uebel, Die Kleruchen, S. $11-13$.

18 žy Ägyptens bis Diokletian, Diss. Freiburg 1965, S. 43 ist es im Gegensatz zur $\chi \varrho \tilde{\eta} \sigma เ \varsigma$ beim $\delta a ́ v \varepsilon \iota v v$, um das es sich hier ja handelt (vgl. Z. 16), ungewöhnlich, daß das Dar-

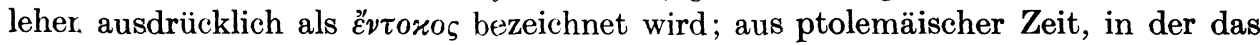
Wort überhaupt recht selten vorkommt, kann Kühnert nur P. Straßb. II 92, 8 (Oxyrhynchites; 244/43 v. Chr.) und P. Oxy. XIV 1644 (Oxyrhynchites; 63/62 v. Chr.) als Beispiele zitieren. Eine Parallele für das hier begegnende Formular ( $\varepsilon^{\prime} v \tau o \varkappa o v$ ohne begleitendes Substantiv und parallel zu $\delta \varrho \alpha \chi \mu \alpha ́ \varsigma$ als Objekt zu $\varepsilon \delta \alpha ́ v \varepsilon 6 \sigma \varepsilon v)$ haben wir überaaupt nicht finden können; immerhin sind P. Hibeh I $89=$ SB XIV 11376, 8 (Thothis; 239/38 v. Chr.) und BGU XIV 2396, 6 (Tholthis; 213/12 v. Chr.) zu ver- 


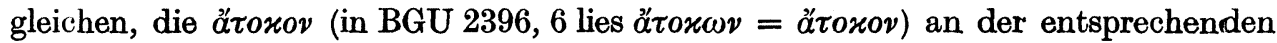
Stelle haben. Vgl. auch F. Uebel in APF 22/23, 1974, 107 zu Z. 5.

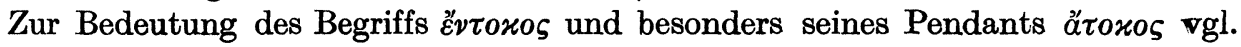
P. W. Pestman, Loans Bearing No Interest?, JJP 16/17, 1971, 7-29. Er macht wahrscheinlich, daß als हैंv während bei den sogenannten ,zinslosen“ Darlehen die vereinbarten Zinsen schon bei der Darlehensgewährung vom Kapital in Abzug gebracht wurden.

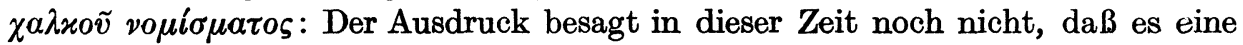
selbständige Kupferwährung neben der Silberwährung gab, sondern nur, daß der Gegenwert von 100 Silberdrachmen in Kupferkleingeld bezahlt worden ist; vgl. dazu z. B. F. Heichelheim in BGU VII S. 274f.; ders., Wirtschaftliche Schwankungen der Zeit von Alexander bis Augustus, Jena 1930, S. 16 f.

18-19 Der Zinssatz von 2\% monatlich, also $25 \%$ oder $24 \%$ jährlich, je nachdem, ob man den makedonischen oder den ägyptischen Kalender zugrundelegt (s. Pestman, JJP 16/17, 1971, 8 Fußn. 4), war in ptolemäischer Zeit der übliche; vgl. H.-A. Rupprecht, Untersuchungen zum Darlehen im Recht der graeco-aegyptischen Papyri der Ptolemäerzeit (Münchener Beiträge 51), München 1967, S. 88f.; Kühnert, Kreditgeschäft, S. 38-44.

21-22 Daß die Rückzahlung des Kapitals nicht zu einem festen Zeitpunkt vereinbart wird, sondern zehn Tage nach der Aufkündigung fällig werden soll, wird auch in folgenden ptolemäischen Darlehensverträgen vereinbart: P. Hibeh I 88, $11 \mathrm{f}$. (Phebichis; 263/62 v. Chr.); APF VII $19=$ SB X 10251, 7 f. (Arsinoites?; 252/51 v. Chr.) und BGU X 1961, 7 (Tholthis; 213/12 v. Chr.). Gründe für diese bemerkenswerte Bestimmung sind nicht ersichtlich. Die früher gelegentlich geäußerte Vermutung, bei solchen Darlehen handele es sich in Wirklichkeit um verschleierte Mitgiften, die zehn Tage nach einer eventuellen Scheidung zurückzuzahlen seien, wird durch den vorliegenden Text, in dem sowohl Gläubiger als auch Schuldner Männer sind, ein weiteres Mal widerlegt; vgl. auch schon Rupprecht, Untersuchungen zum Darlehen, S. 68 und Uebel, Agoranomion (s. oben zu Z. 5), S. 443f. Eine Frist von zehn Tagen erscheint weiterhin in einigen Urkunden über Grundstückserwerb, nämlich in P. Hibeh I 89 = SB XIV 11376, 14 (Tholthis; 239/8 v. Chr.); BGU X 1974 = BGU XIV 2398, 11f. (Tholthis; 213/2 v. Chr.); SB XIV 11375, 14.30 (Tholthis; 211/10 v. Chr.) und BGU X 1973, 2 (Oxyrhynchites; Zeit d. Philopator); zu diesen Texten vgl. ausführlich Uebel, Agoranomion, S. 440-452.

23-24 Ergänzt nach Z. 2-3. Am Anfang von Z. 24 sieht man noch einige sehr schwache Tintenspuren, die sich aber nicht einzelnen Buchstaben zuordnen lassen.

\section{Utbersetzung (Z. 12-24;3-5)}

Unter der Königsherrschaft des Ptolemaios, des Sohnes des Ptolemaios und der Arsinoe, der Geschwister-Götter, im sechzehnten Jahr, zu der Zeit, da Aristanax, der Sohn des Timandros, Priester des Alexander, der Geschwister-Götter und der Wohltäter-Götter ist und Satyra, die Tochter des Artemidoros, Kanephore der Arsinoe Philadelphos ist, im Monat Peritios, nach ägyptischem Kalender Pachons, in Tholthis im Oxyrhynchites.

Es hat ausgeliehen Menandros, Perser, einfacher Soldat aus der Truppe des Zoilos, an Ammonios, den Sohn des Ammonios, - - - von Abstammung, als noch zu ver- 
zinsendes Darlehen einhundert Drachmen in Kupfergeld zu einem Zinssatz von zwei Drachmen pro Mine für jeden Monat. Ammonios soll Menandros die Zinsen in jedem Monat zurückzahlen, das Darlehen aber innerhalb von zehn Tagen, gerechnet von dem Tag an, an welchem Menandros es ansagt. Wenn er aber nicht zurückzahlt, wie es geschrieben steht, soll Ammonios an Menandros als Strafe die Zinsen, die er zusätzlich schuldet, in einfacher Höhe, das Darlehen aber in anderthalbfacher Höhe zahlen, und die Vollstreckung soll Menandros von Ammonios zustehen, wobei er entsprechend dem (königlichen) Erlaß vollstreckt. Diese Abmachung ist maßgeblich, wo auch immer sie vorgewiesen wird. Zeugen sind: (Die Angabe ist unterblieben). 
